\title{
Preparation, Spectral Characterization and Anticancer Potential of Cinnamic Esters
}

\author{
Renan O. Gonçalves, ${ }^{\circledR a}$ Iolanda F. de Farias, ${ }^{a}$ Maria Francilene S. Silva, ${ }^{b}$ \\ Cláudia Ó. Pessoa, ${ }^{b}$ Guilherme J. Zocolo, ${ }^{\circledR c}$ Davila Zampieri, ${ }^{d}$ Telma Leda G. de Lemos ${ }^{a}$ \\ and Francisco Jose $Q$. Monte ${ }^{\circledR *, a}$ \\ ${ }^{a}$ Departamento de Química Orgânica e Inorgânica, Universidade Federal do Ceará, \\ Campus do Pici, 60021-940 Fortaleza-CE, Brazil \\ ${ }^{b}$ Núcleo de Pesquisa e Desenvolvimento de Medicamentos, Universidade Federal do Ceará, \\ Rua Coronel Nunes de Mello, 60420-275 Fortaleza-CE, Brazil \\ ${ }^{c}$ Embrapa Agroindústria Tropical, Rua Dra. Sara Mesquita, Pici, 60020-181 Fortaleza-CE, Brazil \\ ${ }^{d}$ Grupo de Pesquisa em Biotecnologia e Espectrometria de Massas, Universidade Federal do Ceará, \\ 60440-900 Fortaleza-CE, Brazil
}

\begin{abstract}
Cinnamic acid and its derivatives show a remarkable variety of biological activities and are often studied in search of the development of new and highly effective drugs. This work aims to synthesize, characterize and evaluate the cytotoxic activity of esters derived from cinnamic acid. Eighteen esters were synthesized through Steglich's esterification, of which eleven were not reported in the literature. All compounds were fully characterized by Fourier transform infrared epectroscopy (FTIR), nuclear magnetic resonance $\left({ }^{1} \mathrm{H}\right.$ and $\left.{ }^{13} \mathrm{C} \mathrm{NMR}\right)$ and high-resolution mass spectrometry (HRMS) data. The cytotoxic activity of esters obtained was evaluated using four human tumor cell lines: SNB-19 (astrocytoma), HCT-116 (colon carcinoma, human), PC3 (prostate) and HL60 (promyelocytic leukemia) through the 3-(4,5-dimethyl-2-thiazolyl)2,5-diphenyl-2H-tetrazolium (MTT) colorimetric assay. These studies showed that the compound 3-methoxybenzyl (E)-3-(4-methoxyphenyl)acrylate (12) is the most potent against HCT-116, PC3 and SBN-19 cells, with the lowest half maximal inhibitory concentration $\left(\mathrm{IC}_{50}\right)$ value of $16.2 \mu \mathrm{M}$ in the HCT-116 strain. The derivatives were obtained in good yields (76.6-95\%), except for compounds 5-isopropyl-2-methylphenyl (E)-3-(3-hydroxy-4-methoxyphenyl)acrylate (17) (18.6\%) and 2-isopropyl-5-methylphenyl (E)-3-(3-hydroxy-4-methoxyphenyl)acrylate (18) (15.5\%).
\end{abstract}

Keywords: cinnamic esters, cytotoxicity, spectral data, Steglich esterification

\section{Introduction}

Among the countless diseases that affect humanity, cancer is that one that affects millions of people, being the second cause of death worldwide, with an estimated number of 9.6 million in the year of $2018 .{ }^{1}$ Treatment of the disease includes chemotherapy, which uses drugs that destroy cancer cells. Several chemotherapeutic agents are used such as doxorubicin, epirubicin and cyclophosphamide, among many others. However, these chemical agents often cause serious side effects. ${ }^{2}$ In this context, numerous researches around the world are related to the development of new drugs to fighting cancer, many of them related to works involving derivatives of natural products. ${ }^{3,4}$ In addition,

*e-mail: fmonte@dqoi.ufc.br cinnamic acid and similar such as acids caffeic and ferulic, are important nutrients present in human food. Several food-stuffs (coffee, chocolate, almonds, among others) that are part of the diet of many people are potentially rich of this type of constituents. ${ }^{5,6}$

There are reports in the literature on the cytotoxic activity of cinnamic acid (1a) and some of its analogs: acid $p$-methoxycinnamic (2a), ferulic acid (3a), isoferulic acid (4a), $p$-hydroxycinnamic acid (5a) and caffeic acid (6a), Figure 1, against some cancer cell lines: MCF-7 (breast carcinoma), PC3 (prostate) and SW480 (human colon). ${ }^{7}$ It is worth mentioning that $\mathbf{1 a}$ has attracted the attention of researchers for a long time, due to its anti-cancer properties. ${ }^{6}$

Research has shown that synthetic derivatives of phenylpropanoid acids have several biological activities: 
<smiles>O=C(O)/C=C/c1ccccc1</smiles><smiles>COc1ccc(/C=C/C(=O)O)cc1</smiles><smiles>COc1cc(/C=C/C(=O)O)ccc1O</smiles><smiles>COc1ccc(/C=C/C(=O)O)cc1O</smiles>

Figure 1. Compounds belonging to the family of phenylpropanoid acids.

hypolipidemic,${ }^{8}$ hypoglycemic, ${ }^{9}$ acetylcholinesterase inhibitor, ${ }^{10}$ antioxidant, ${ }^{11}$ antimicrobial, ${ }^{12}$ antimalarial, ${ }^{13}$ antifungal, ${ }^{14}$ and anticancer. ${ }^{15}$ Others studies have also revealed anticancer properties of cinnamates in specific human tumor cell lines: HeLa 127 (cervix), MCF-7 (breast), PC3 (prostate) and K562 (myeloid leukemia). ${ }^{15,16}$ In these studies, cinnamates had shown promising anticancer activity, presenting a high level of cytotoxicity and selectivity.

An effective and simple method for forming cinnamates is to use the esterification of Steglich, ${ }^{17,18}$ for not wanting high temperatures or using acyl halide, and in milder reaction media and forming very reactive intermediates, being possible to apply to different reaction systems. The Steglich reaction is a modification of an esterification in which $N, N^{\prime}$-dicyclohexylcarbodiimide (DCC) acts as a coupling reagent and 4-( $N, N^{\prime}$-dimethylamino)pyridine (DMAP) as a catalyst. Initially, the DCC acts as a base and gives rise to the carboxylate anion which, in turn, attacks the protonated DCC imidic carbon forming the $O$-acylisourea. This, with reactivity similar to acid anhydride, is protonated, indirectly activating its carbonyl carbon to attack the hydroxyl group of alcohol. After deprotonation, precipitation of dicyclohexylurea (DCU) occurs with formation of the ester (Figure 2). ${ }^{19}$

In order to evaluate the anticancer activity of cinnamates, the present work describes the synthesis via esterification of Steglish and characterization of esters of cinnamic acid, $p$-methoxycinnamic acid and ferulic acid. The cytotoxic activity of esters (1-18) was assessed using four human tumor cell lines: SNB-19 (astrocytoma), HCT-116 (colon carcinoma, human), PC3 (prostate) and HL60 (promyelocytic leukemia), in addition to of a healthy L929 cell (murine fibroblast).

\section{Experimental}

\section{Chemistry}

The reagents acetic anhydride, triethylamine and dihydrocarvenol were obtained from Vetec (Caxias do Sul, Brazil). Additionally, potassium bromide $(\mathrm{KBr})$, deuterochloroform $\left(\mathrm{CDCl}_{3}\right)$, doxorubicin hydrochloride, 3-(4,5-dimethyl-2-thiazolyl)-2,5-diphenyl$2 H$-tetrazolium bromide (MTT), cinnamic acid, 4-methoxy-cinnamic acid, 3-methoxy-4-hydroxycinnamic acid, $(S)$-(-)-perillyl alcohol, carvacrol, thymol, 5-indanol, 6-hydroxy-1 $H$-isocromen-1-one, vanillin, $N, N^{\prime}$-dicyclohexylcarbodiimide (DCC) and 4-( $N, N$ '-dimethylamino)pyridine (DMAP), were obtained from Sigma-Aldrich Corporation (Saint Louis, USA). The solvents dichloromethane, dimethyl sulfoxide (DMSO), hexane and ethyl acetate, were obtained from Synth (Diadema, Brazil). Nuclear magnetic resonance
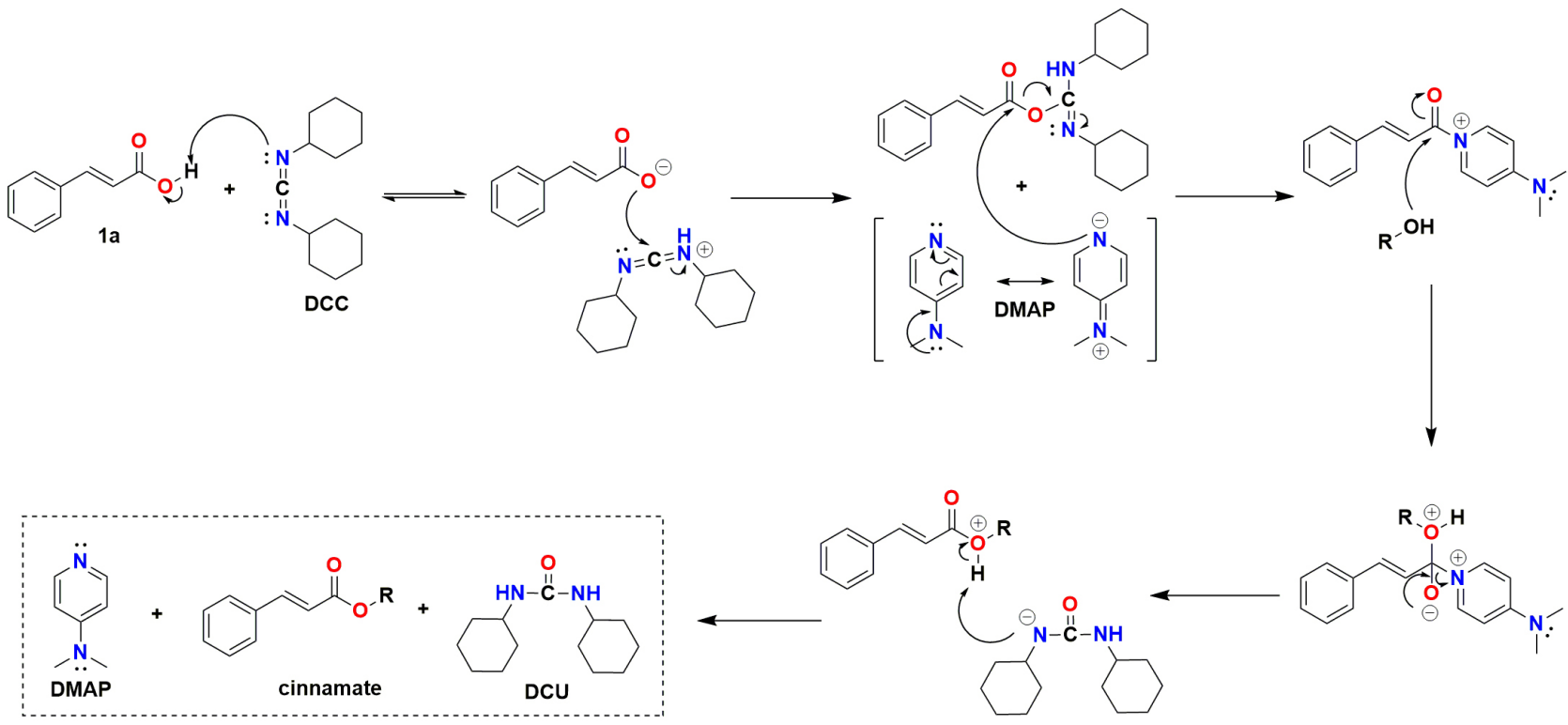

Figure 2. Reaction mechanism of Steglish esterification. 
(NMR) spectra were recorded on a Bruker Avance DPX-300 NMR spectrometer $\left(300 \mathrm{MHz}\right.$ for ${ }^{1} \mathrm{H}$ and $75 \mathrm{MHz}$ for ${ }^{13} \mathrm{C}$ ) (Washington, USA) using $\mathrm{CDCl}_{3}$ solutions, and all chemical shifts reported in $\mathrm{ppm}(\delta$ units) with residual $\mathrm{CHCl}_{3}(\delta$ 7.27) as internal standard for ${ }^{1} \mathrm{H}$ NMR and the central peak of the triplet $(\delta 77.23)$ of $\mathrm{CDCl}_{3}$ for ${ }^{13} \mathrm{C}$ NMR. Infrared (IR) spectra were taken as $\mathrm{KBr}$ pellets, on PerkinElmer spectrophotometer, model FTIR SPECTRUM (Ontario, Canada). High-resolution mass spectrometry (HRMS) were obtained on XEVO TQ-D triple quadrupole mass spectrometer coupled to a MassLynxTM software (Santa Clara, USA); samples were introduced into the system by direct infusion, being ionized by electrospray operating in positive ion mode [ESI(+)]. Flash chromatography columns were performed using silica gel $60(0.040-0.063 \mathrm{~mm})$ purchased from Merck (Darmstadt, Germany), adapted to the pressure system with an Omron NE-C 801 compressor (São Paulo, Brazil); reactions were monitored by analytical thin layer chromatography (TLC) utilizing aluminium silica gel $60 \mathrm{~F} 254$ precoated $0.25 \mathrm{~nm}$ plates, from the same manufacturer, with visualization under UV light $(254 \mathrm{~nm})$. Melting points were determined in Mettler Toledo digital micro determination equipment and are uncorrected (Ohio, USA). All yields reported refer to isolated yields.
General procedure for the synthesis of compounds 1-8

In separate experiments, cinnamic acid (1a) (60.0 $\mathrm{mg}, 0.44 \mathrm{mmol}, 1$ equiv) in dichloromethane solution $(5 \mathrm{~mL})$ under stirring was mixed with $N, N^{\prime}$ dicyclohexylcarbodiimide (DCC) $(90.7 \mathrm{mg}, 0.44 \mathrm{mmol}$, 1.1 equiv) and 4 -( $N, N^{\prime}$-dimethylamino)pyridine (DMAP) (48.80 mg, $0.44 \mathrm{mmol}, 1$ equiv). In each mixture, the corresponding alcohol/phenol $(0.44,1.1$ equiv) was added, followed by stirring of the resulting solutions at room temperature for $24 \mathrm{~h}$ (Scheme 1). At the end of each reaction, the solutions were filtered and concentrated under reduced pressure. The crude products were purified on silica gel chromatographic columns eluted with 9:1 hexane/EtOAc.

\section{General procedure for the synthesis of compounds 9-16}

In separate experiments, $p$-methoxycinnamic acid (2a) (60.0 mg, $0.33 \mathrm{mmol}, 1$ equiv) in dichloromethane solution $(7 \mathrm{~mL})$ under stirring was mixed with $N, N^{\prime}$-dicyclohexylcarbodiimide (DCC) $(69.5 \mathrm{mg}, 0.33 \mathrm{mmol}$, 1.1 equiv) and 4-( $N, N^{\prime}$-dimethylamino)pyridine (DMAP) (40.90 $\mathrm{mg}, 0.33 \mathrm{mmol}, 1$ equiv). In each mixture, the corresponding alcohol or phenol ( $0.33 \mathrm{mmol}, 1.1$ equiv)

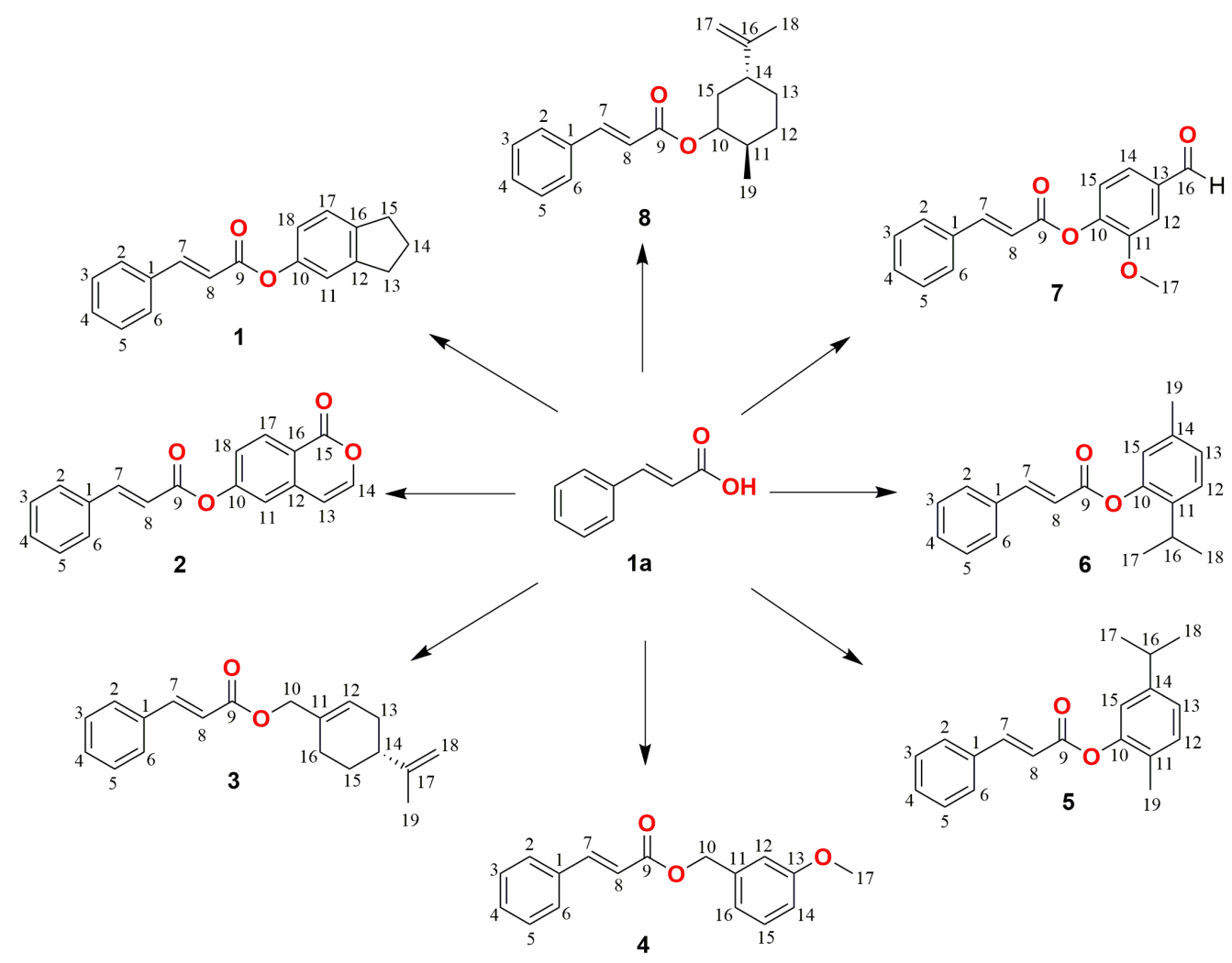

Scheme 1. Synthesis of cinnamic acid derivatives; alcoholic/phenolic reagents, DCC, DMAP, $\mathrm{CH}_{2} \mathrm{Cl}_{2}, \mathrm{rt}, 24 \mathrm{~h}$. 
was added, followed by stirring of the resulting solutions at room temperature for $24 \mathrm{~h}$ (Scheme 2). At the end of each reaction, the solutions were filtered and concentrated under reduced pressure. The crude products were purified on silica gel chromatographic columns eluted with 8:2 hexane/EtOAc.

\section{General procedure for the synthesis of compounds 17 and} 18

4-(N,N'-Dimethylamino)pyridine (DMAP) $(53.7 \mathrm{mg}$, $0.44 \mathrm{mmol}, 1$ equiv $)$ and acetic anhydride $\left(\mathrm{Ac}_{2} \mathrm{O}\right)(415 \mu \mathrm{L}$, $4.4 \mathrm{mmol}, 10$ equiv) in dichloromethane $(3 \mathrm{~mL})$ were mixed and stirred by $5 \mathrm{~min}$ at room temperature. Then, ferulic acid (3a) (87.1 mg, $0.44 \mathrm{mmol}, 1$ equiv) and triethylamine $\left(\mathrm{Et}_{3} \mathrm{~N}\right)(30.5 \mu \mathrm{L}, 0.22 \mathrm{mmol}, 0.5$ equiv) were added and the mixture was stirred for another $30 \mathrm{~min}$. Next, the solution was concentrated under reduced pressure. The crude product was precipitated by adding $5 \mathrm{~mL}$ of ice water and filtered under vacuum. The $\mathbf{3 b}$ product was obtained with $86.3 \%$ (91.3 mg) yield. Then compound $\mathbf{3 b}$ (96 mg, $0.40 \mathrm{mmol}, 1$ equiv) was added to a stirred solution in dichloromethane (5 mL), DCC (90.6 mg, $0.44 \mathrm{mmol}$, 1.1 equiv) and DMAP (48.8 mg, $0.44 \mathrm{mmol}, 1$ equiv). Soon after, appropriate phenol ( $0.44 \mathrm{mmol}, 1.1$ equiv) was added and the mixture was stirred for $8 \mathrm{~h}$ at room temperature (Scheme 3). At the end of the reaction, the residue was concentrated under reduced pressure. The crude product was purified by column chromatography with silica gel, eluting with hexane/EtOAc (9:1 v/v), obtaining the products $\mathbf{1 7}$ and $\mathbf{1 8}$.

\section{2,3-Dihydro- $1 H$-inden-5-yl cinnamate (1)}

Following the general procedure, and using 5-indanol (59 mg, $0.44 \mathrm{mmol}$ ), compound 1 was obtained as white solid in 95\% yield; $\mathrm{mp} 79-81{ }^{\circ} \mathrm{C}$; IR $(\mathrm{KBr}) \mathrm{v} / \mathrm{cm}^{-1} 3062$, 2929, 2845, 1728, 1631, 1481, 1309, 1143, 989, 871, 763, 680; ${ }^{1} \mathrm{H} \mathrm{NMR}\left(300 \mathrm{MHz}, \mathrm{CDCl}_{3}\right) \delta 7.88(\mathrm{~d}, 1 \mathrm{H}, J 16.0 \mathrm{~Hz}$, H-7), 7.60 (m, 2H, H-2, H-6), 7.43 (m, 3H, H-3, H-4, H-5), 7.24 (d, 1H, J $8.1 \mathrm{~Hz}, \mathrm{H}-17$ ), 7.03 (brs, 1H, H-11), 6.92 (dd, $1 \mathrm{H}, J 8.0,2.1 \mathrm{~Hz}, \mathrm{H}-18), 6.65$ (d, 1H, J 16.0 Hz, H-8), 2.93 (brq, 4H, 2H-13, 2H-15), 2.21 (brq, 2H, 2H-14); ${ }^{13} \mathrm{C} \mathrm{NMR}$ $\left(75 \mathrm{MHz} \mathrm{CDCl}_{3}\right) \delta 166.0$ (C-9), 149.5 (C-10), 146.4 (C-7), 145.9 (C-12), 141.9 (C-16), 134.5 (C-1), 130.8 (C-4), 129.1 (C-2, C-6), 128.4 (C-3, C-5), 125.0 (C-17), 119.3 (C-18), $117.8(\mathrm{C}-11)^{*}, 117.7(\mathrm{C}-8) *, 33.2$ (C-13), 32.5 (C-15), 25.9 (C-14), *exchangeable assignments; HRMS $m / z$, calcd. for $\mathrm{C}_{18} \mathrm{H}_{16} \mathrm{O}_{2}[\mathrm{M}+\mathrm{H}]^{+}:$265.1150, found: 265.1922.

\section{1-Oxo-1H-isochromen-6-yl cinnamate (2)}

Following the general procedure, and using 6-hydroxy$1 \mathrm{H}$-isocromen-1-one (71.3 mg, $0.44 \mathrm{mmol})$, compound 2 was obtained as white solid in $91.4 \%$ yield; $\mathrm{mp} 150-152{ }^{\circ} \mathrm{C}$;

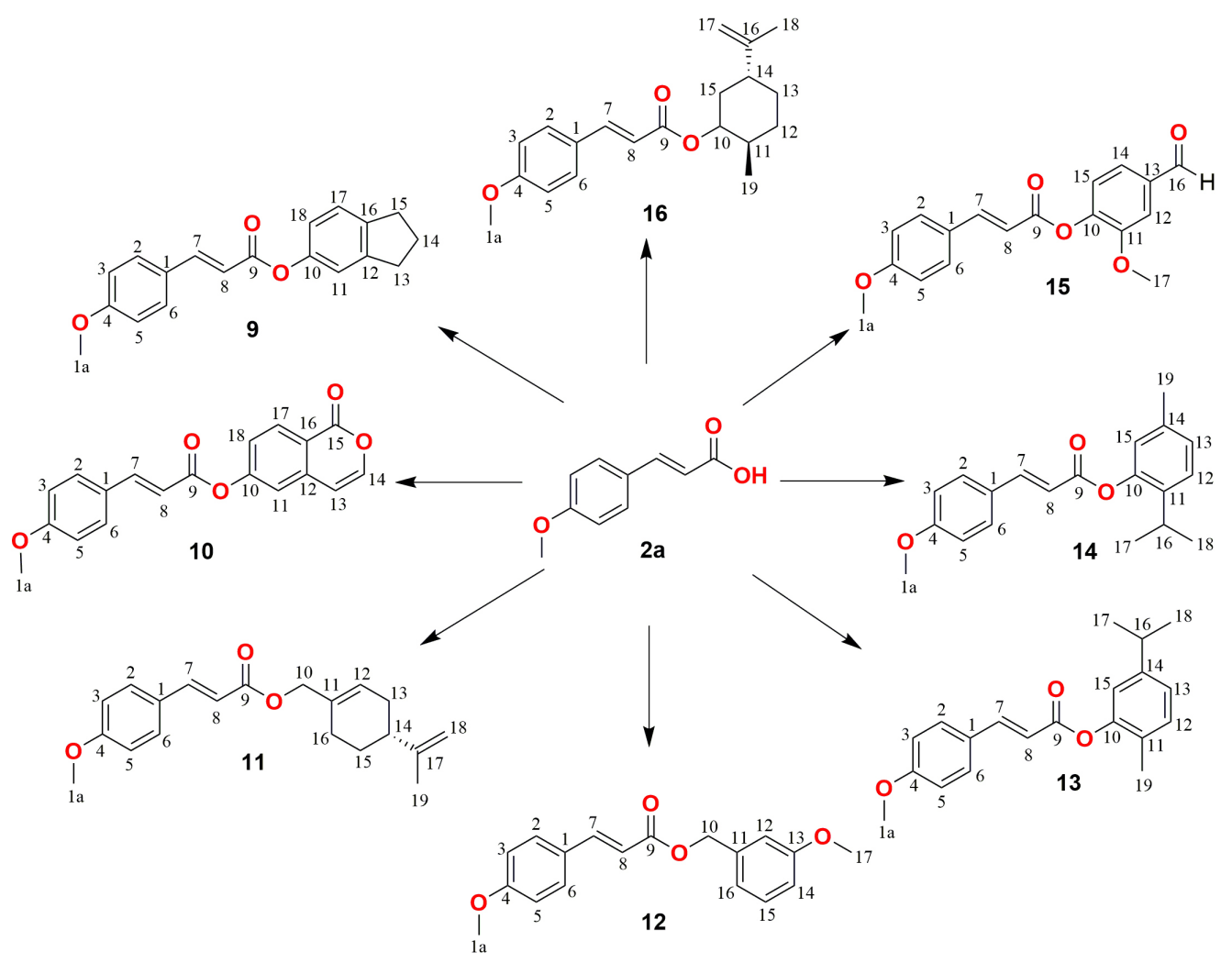

Scheme 2. Synthesis of $p$-methoxycinnamic acid derivatives; alcoholic/phenolic reagents, DCC, DMAP, $\mathrm{CH}_{2} \mathrm{Cl}_{2}$, reflux, $6 \mathrm{~h}$. 


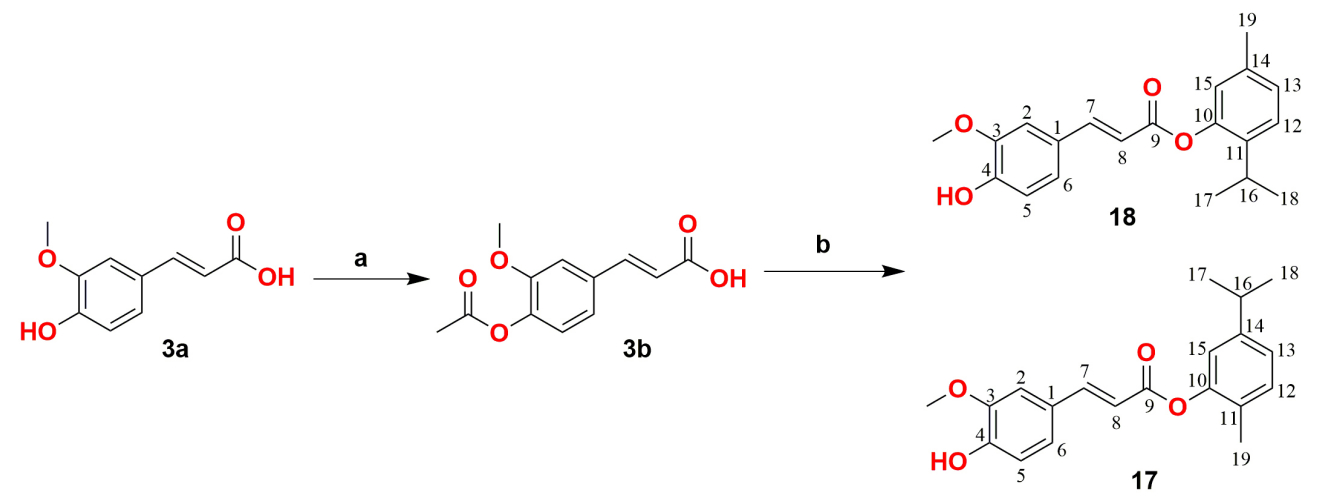

Scheme 3. Synthesis of ferulic acid derivatives; (a) $\mathrm{Et}_{3} \mathrm{~N}$, DMAP, $\mathrm{Ac}_{2} \mathrm{O}, \mathrm{CH}_{2} \mathrm{Cl}_{2}, \mathrm{rt}, 30 \mathrm{~min}$; (b) phenolic reagent, DCC, DMAP, $\mathrm{CH}_{2} \mathrm{Cl}_{2}, \mathrm{rt}, 8 \mathrm{~h}$.

IR (KBr) v / $\mathrm{cm}^{-1} 3080,3053,1728,1629,1307,1263$, $1132,856,759 ;{ }^{1} \mathrm{H}$ NMR $\left(300 \mathrm{MHz}, \mathrm{CDCl}_{3}\right) \delta 7.91(\mathrm{~d}, 1 \mathrm{H}$, $J 16.0 \mathrm{~Hz}, \mathrm{H}-7), 7.72(\mathrm{~d}, 1 \mathrm{H}, J 9.6 \mathrm{~Hz}, \mathrm{H}-14), 7.65-7.58$ (m, 2H, H-2, H-6), 7.52 (d, 1H, J 8.4 Hz, H-17), 7.48-7.42 (m, 3H, H-3, H-4, H-5), 7.22 (d, 1H, J $1.6 \mathrm{~Hz}, \mathrm{H}-11$ ), 7.16 (dd, $1 \mathrm{H}, J$ 8.4, 2.0 Hz, H-18), 6.64 (d, $1 \mathrm{H}, J 16.0 \mathrm{~Hz}$, $\mathrm{H}-8), 6.41(\mathrm{~d}, 1 \mathrm{H}, J 9.6 \mathrm{~Hz}, \mathrm{H}-13) ;{ }^{13} \mathrm{C}$ NMR $(75 \mathrm{MHz}$, $\mathrm{CDCl}_{3}$ ) $\delta 164.7$ (C-9), 160.4 (C-15), 154.7 (C-10), 153.4 (C-12), 147.7 (C-7), 142.9 (C-14), 133.9 (C-1), 131.1 (C-4), 129.1 (C-2, C-6), 128.6 (C-17), 128.5 (C-3, C-5), 118.5 (C-8), 116.7 (C-16), 116.5 (C-11)*, 116.1 (C-18)*, 118.5 (C-18), 110.5 (C-13), *exchangeable assignments; HRMS $m / z$, calcd. for $\mathrm{C}_{18} \mathrm{H}_{12} \mathrm{O}_{4}[\mathrm{M}+\mathrm{H}]^{+}:$293.0814, found: 293.0818 .

(S)-(4-(Prop-1-en-2-yl)cyclohex-1-en-1-yl)methyl cinnamate (3)

Following the general procedure, and using (S)-(-)-perillyl alcohol (70 $\mu \mathrm{L}, 0.44 \mathrm{mmol})$, compound 3 was obtained as yellow liquid in $88.4 \%$ yield; IR $(\mathrm{KBr})$ $v / \mathrm{cm}^{-1} 3062,2924,2117,1712,1639,1450,1307,1165$, 979, 767; ${ }^{1} \mathrm{H}$ NMR $\left(300 \mathrm{MHz}, \mathrm{CDCl}_{3}\right) \delta 7.71(\mathrm{~d}, 1 \mathrm{H}$, $J 16.0 \mathrm{~Hz}, \mathrm{H}-7$ ), 7.53 (m, 2H, H-2, H-6), 7.39 (m, 3H, H-3, $\mathrm{H}-4, \mathrm{H}-5), 6.48$ (d, 1H, J 16.0 Hz, H-8), 5.83 (m, 1H, H-12), 4.74 (d, 2H, J3.0 Hz, 2H-18), 4.62 (s, 2H, 2H-10), 2.20 (m, 3H, H-16, H-14, H-13), 2.00 (m, 2H, H-16', H-13'), 1.89 (m, 1H, H-15), 1.75 (s, 3H, 3H-17), 1.54 (m, 1H, H-15'); ${ }^{13} \mathrm{C}$ NMR $\left(75 \mathrm{MHz}, \mathrm{CDCl}_{3}\right.$ ) $\delta 167.1$ (C-9), $149.8(\mathrm{C}-17)$, 145.0 (C-7), 134.6 (C-1), 132.9 (C-11), 130.4 (C-4), 129.0 (C-2, C-6), 128.2 (C-3, C-5), 126.0 (C-12), 118.3 (C-8), 108.9 (C-18), 68.7 (C-10), 41.0 (C-14), 30.7 (C-16), 27.5 (C-13), 26.6 (C-15), 20.9 (C-19); HRMS $\mathrm{m} / \mathrm{z}$, calcd. for $\mathrm{C}_{19} \mathrm{H}_{22} \mathrm{O}_{2}[\mathrm{M}+\mathrm{H}]^{+}: 283.1698$, found: 283.1698 .

\section{3-Methoxyphenyl cinnamate (4)}

Following the general procedure, and using 3-methoxybenzyl alcohol ( $61 \mu \mathrm{L}, 0.44 \mathrm{mmol})$, compound 4 was obtained as yellow liquid in $83.5 \%$ yield; IR $(\mathrm{KBr})$ $v / \mathrm{cm}^{-1} 2954,2835,1712,1635,1600,1490,1450,1311$, 1269, 1163, 979, 767, 686; ${ }^{1} \mathrm{H}$ NMR (300 MHz, $\mathrm{CDCl}_{3}$ ) $\delta 7.70(\mathrm{~d}, 1 \mathrm{H}, J 16.0 \mathrm{~Hz}, \mathrm{H}-7), 7.49$ (m, 2H, H-2, H-6), 7.35 (m, 3H, H-3, H-4, H-5), 7.26 (t, 1H, J 7.9 Hz, H-15), 6.96 (brd, $1 \mathrm{H}, J 8.2 \mathrm{~Hz}, \mathrm{H}-16$ ), 6.93 (brs, $1 \mathrm{H}, \mathrm{H}-12$ ), 6.85 (dd, $1 \mathrm{H}, J 8.2,2.3 \mathrm{~Hz}, \mathrm{H}-14), 6.48$ (d, $1 \mathrm{H}, J 16.0 \mathrm{~Hz}, \mathrm{H}-8$ ), 5.20 (s, 2H, 2H-10), 3.79 (s, 3H, $\left.\mathrm{CH}_{3} \mathrm{O}-17\right) ;{ }^{13} \mathrm{C}$ NMR $\left(75 \mathrm{MHz}, \mathrm{CDCl}_{3}\right) \delta 166.9(\mathrm{C}-9), 160.0(\mathrm{C}-13), 145.4(\mathrm{C}-7)$, 137.7 (C-11), 134.5 (C-1), 130.5 (C-4), 129.8 (C-15), 129.1 (C-2, C-6), 128.3 (C-3, C-5), 120.6 (C-16), 118.0 (C-8), $114.0(\mathrm{C}-14)^{*}, 113.9 *$ (C-12), 66.4 (C-10), 55.4 $\left(\mathrm{CH}_{3} \mathrm{O}-17\right)$, *exchangeable assignments; HRMS $\mathrm{m} / z$, calcd. for $\mathrm{C}_{17} \mathrm{H}_{16} \mathrm{O}_{3}[\mathrm{M}+\mathrm{H}]^{+}:$269.1178, found: 269.0663. Data are in agreement with those previously reported. ${ }^{17}$

\section{2-Isopropyl-5-methylphenyl cinnamate (5)}

Following the general procedure, and using thymol ( $66 \mathrm{mg}, 0.44 \mathrm{mmol}$ ), compound 5 was obtained as yellow viscose liquid in 97\% yield; $\mathrm{IR}(\mathrm{KBr}) v / \mathrm{cm}^{-1} 3059,2962$, 2926, 2868, 1726, 1639, 1506, 1448, 1309, 1244, 1161, 981, 765; ${ }^{1} \mathrm{H}$ NMR $\left(300 \mathrm{MHz}, \mathrm{CDCl}_{3}\right) \delta 7.91(\mathrm{~d}, 1 \mathrm{H}$, $J 16.0 \mathrm{~Hz}, \mathrm{H}-7), 7.63$ (m, 2H, H-2, H-6), 7.45 (m, 3H, H-3, H-4, H-5), 7.26 (d, 1H, J 8.0 Hz, H-12), 7.21 (brd, $1 \mathrm{H}, J 7.9 \mathrm{~Hz}, \mathrm{H}-13$ ), 6.92 (brs, 1H, H-15), 6.70 (d, 1H, $J 16.0 \mathrm{~Hz}, \mathrm{H}-8$ ), 3.07 (q, 1H, J 6.0 Hz, H-16), 2.36 (s, 3H, $3 \mathrm{H}-19), 1.24$ (d, 6H, J 6.0 Hz, 3H-17, 3H-18); ${ }^{13} \mathrm{C}$ NMR $\left(75 \mathrm{MHz}, \mathrm{CDCl}_{3}\right) \delta 165.9(\mathrm{C}-9), 148.1$ (C-10), $146.6(\mathrm{C}-7)$, 137.3 (C-14), 136.7 (C-11), 134.4 (C-1), 130.8 (C-4), 129.1 (C-2, C-6), 128.5 (C-3, C-5), 127.3 (C-12), 126.6 (C-13), 123.0 (C-15), 117.5 (C-8), 27.3 (C-16), 23.2 (C-17, C-18), 21.0 (C-19); HRMS $m / z$, calcd. for $\mathrm{C}_{19} \mathrm{H}_{20} \mathrm{O}_{2}[\mathrm{M}+\mathrm{H}]^{+}$: 281.1542, found: 281.1563. Data are in agreement with those previously reported. ${ }^{20}$

\section{5-Isopropyl-2-methylphenyl cinnamate (6)}

Following the general procedure, and using carvacrol (64 $\mu \mathrm{L}, 0.44 \mathrm{mmol}$ ), compound 6 was obtained as white solid in $93.6 \%$ yield; mp $45-46{ }^{\circ} \mathrm{C}$; IR $(\mathrm{KBr}) \vee / \mathrm{cm}^{-1}$ 
2964, 2872, 1728, 1635, 1450, 1236, 1159, 765; ${ }^{1} \mathrm{H}$ NMR $\left(300 \mathrm{MHz}, \mathrm{CDCl}_{3}\right) \delta 7.91(\mathrm{~d}, 1 \mathrm{H}, J 16.0 \mathrm{~Hz}, \mathrm{H}-7), 7.62$ (m, 2H, H-2, H-6), 7.44 (m, 3H, H-3, H-4, H-5), 7.21 (d, $1 \mathrm{H}, J 7.8 \mathrm{~Hz}, \mathrm{H}-12), 7.12$ (dl, 1H, J 7.8 Hz, H-13), 6.97 (brs, 1H, H-15), 6.69 (d, 1H, J 16.0 Hz, H-8), 2.91 (q, 1H, $J 6.9 \mathrm{~Hz}, \mathrm{H}-16), 2.20$ (s, 3H, 3H-19), 1.27 (d, 6H, J 6.9 Hz, $3 \mathrm{H}-17,3 \mathrm{H}-18) ;{ }^{13} \mathrm{C}$ NMR $\left(75 \mathrm{MHz}, \mathrm{CDCl}_{3}\right.$ ) $\delta 165.4$ (C-9), 149.5 (C-10), 148.3 (C-14), 146.6 (C-7), 134.4 (C-1), 131.1 (C-12), 130.8 (C-4), 129.2 (C-2, C-6), 128.5 (C-3, C-5), 127.5 (C-11), 124.3 (C-13), 120.0 (C-15), 117.8 (C-8), 33.8 (C-16), 24.1 (C-17, C-18), 16.0 (C-19); HRMS m/z, calcd. for $\mathrm{C}_{19} \mathrm{H}_{20} \mathrm{O}_{2}[\mathrm{M}+\mathrm{H}]^{+}:$281.2770, found: 281.1542. The structure of compound $\mathbf{6}$ is reported in the literature, ${ }^{21,22}$ but it does not report spectroscopic data.

\section{2-Ethyl-4-formylphenyl cinnamate (7)}

Following the general procedure, and using vanillin ( $66.9 \mathrm{mg}, 0.44 \mathrm{mmol}$ ), compound 7 was obtained as solid white in $87.6 \%$ yield; $\mathrm{mp} 90-92{ }^{\circ} \mathrm{C}$; $\mathrm{IR}(\mathrm{KBr}) \mathrm{v} / \mathrm{cm}^{-1} 3064$, 2927, 2837, 2739, 1734, 1701, 1633, 1597, 1502, 1425, $1269,1139,1118,761 ;{ }^{1} \mathrm{H}$ NMR $\left(300 \mathrm{MHz}, \mathrm{CDCl}_{3}\right) \delta 9.98$ (s, 1H, H-16), 7.91 (d, 1H, J 16.0 Hz, H-7), 7.63-7.59 (m, 2H, H-2, H-6), 7.54 (brs, 1H, H-12), 7.52 (brdd, 1H, J 8.0, $3.0 \mathrm{~Hz}, \mathrm{H}-14), 7.44$ (m, 3H, H-3, H-4, H-5), 7.32 (d, 1H, $J 7.8 \mathrm{~Hz}, \mathrm{H}-15), 6.68$ (d, 1H, J 16.0 Hz, H-8), 3.92 (s, 3H, $\left.\mathrm{CH}_{3} \mathrm{O}-17\right) ;{ }^{13} \mathrm{C}$ NMR $\left(75 \mathrm{MHz}, \mathrm{CDCl}_{3}\right) \delta 191.2(\mathrm{C}-16)$, 164.4 (C-9), 152.2 (C-10), 147.4 (C-7), 145.4 (C-11), 135.2 (C-13), 134.1 (C-1), 130.9 (C-4), 129.2 (C-2, C-6), 128.5 (C-3, C-5), 124.8 (C-14), 123.6 (C-15), 116.5 (C-8), 110.9 (C-12), $56.1\left(\mathrm{CH}_{3} \mathrm{O}-17\right)$; HRMS $\mathrm{m} / \mathrm{z}$, calcd. for $\mathrm{C}_{17} \mathrm{H}_{16} \mathrm{O}_{4}[\mathrm{M}+\mathrm{H}]^{+}: 283.0961$, found: 283.0892. Data are in agreement with those previously reported. ${ }^{23}$

rac-(2S)-2-Methyl-5-(prop-1-en-2-yl)cyclohexyl cinnamate (8)

Following the general procedure, and using racdihydrocarveol (63 $\mu \mathrm{L}, 0.44 \mathrm{mmol})$, compound 8 was obtained as translucent liquid in $79.5 \%$ yield; IR (KBr) $v / \mathrm{cm}^{-1}$ 3066, 2929, 2858, 1708, 1637, 1452, 1307, 1172, 1006, 766; ${ }^{1} \mathrm{H}$ NMR $\left(300 \mathrm{MHz}, \mathrm{CDCl}_{3}\right) \delta 7.70(\mathrm{~d}, 1 \mathrm{H}$, $J$ 16.0 Hz, H-7), 7.54 (m, 2H, H-2, H-6), 7.39 (m, 3H, H-3, H-4, H-5), 6.46 (d, 1H, J 16.0 Hz, H-8), 4.71 (brs, $2 \mathrm{H}, 2 \mathrm{H}-17), 4.64$ (td, 1H, J 10.7, $4.1 \mathrm{~Hz}, \mathrm{H}-10), 2.15$ (m, 2H, H-14, H-15), 1.85 (m, 1H, H-15'), 1.75 (m, 1H, H-11), 1.64 (m, 1H, H-13), 1.65 (s, 3H, 3H-18), 1.38-1.19 (m, 3H, 2H-12, H-13'), 0.97 (d, 3H, J $6.0 \mathrm{~Hz}, 3 \mathrm{H}-19)$; ${ }^{13} \mathrm{C}$ NMR $\left(75 \mathrm{MHz}, \mathrm{CDCl}_{3}\right) \delta 166.9$ (C-9), 149.1 (C-16), 144.6 (C-7), 134.7 (C-1), 130.3 (C-4), 129.0 (C-2, C-6), 128.2 (C-3, C-5), 118.8 (C-8), 109.0 (C-17), 78.5 (C-10), 43.9 (C-11), 37.5 (C-14), 37.2 (C-15), 33.3 (C-12), 31.1 (C-13), 21.1 (C-18), 18.5 (C-19); HRMS $m / z$, calcd. for $\mathrm{C}_{19} \mathrm{H}_{24} \mathrm{O}_{2}$ $[\mathrm{M}+\mathrm{H}]^{+}:$285.1855, found: 284.9321 .
2,3-Dihydro-1H-inden-5-yl (E)-3-(4-methoxyphenyl)acrylate (9)

Following the general procedure, and using 5-indanol (44.3 mg, $0.33 \mathrm{mmol}$ ), compound 9 was obtained as amorphous white solid in $76.6 \%$ yield; $\mathrm{mp} 74.0-76^{\circ} \mathrm{C}$; IR $(\mathrm{KBr}) v / \mathrm{cm}^{-1} 3008,2935,2841,2112,2042,1894,1716$, 1629, 1600, 1510, 1479, 1249, 1145, 993, 823, 549, 414; ${ }^{1} \mathrm{H} \mathrm{NMR}\left(300 \mathrm{MHz}, \mathrm{CDCl}_{3}\right) \delta 7.83(\mathrm{~d}, 1 \mathrm{H}, J 15.9 \mathrm{~Hz}, \mathrm{H}-7)$, 7.55 (d, 2H, J 8.7 Hz, H-2, H-6), $7.23(\mathrm{~d}, 1 \mathrm{H}, J 8.8 \mathrm{~Hz}$, H-17), 7.02 (brs, 1H, H-11), 6.95 (d, 2H, J $8.8 \mathrm{~Hz}, \mathrm{H}-3$, H-5), 6.91* (dd, 1H, J 8.7, $2.5 \mathrm{~Hz}, \mathrm{H}-18), 6.51$ (d, 1H, $J 15.9 \mathrm{~Hz}, \mathrm{H}-8$ ), 3.87 (s, 3H, $\left.\mathrm{CH}_{3} \mathrm{O}-1 \mathrm{a}\right), 2.92$ (q, 4H, 2H-13, $2 \mathrm{H}-15), 2.12$ (m, 2H, 2H-14); ${ }^{13} \mathrm{C} \mathrm{NMR}\left(75 \mathrm{MHz}, \mathrm{CDCl}_{3}\right.$ ) $\delta 166.3$ (C-9), 161.8 (C-4), 149.6 (C-10), 146.1 (C-7), 145.9 (C-12), 141.7 (C-16), 130.1 (C-2, C-6), 127.2 (C-1), 124.9 (C-17), 119.4 (C-18), 117.9 (C-11), 115.1 (C-8), 114.6 (C-3, C-5), $55.6\left(\mathrm{CH}_{3} \mathrm{O}-1 \mathrm{a}\right), 33.2(\mathrm{C}-13)^{*}, 32.5(\mathrm{C}-15)^{*}$, $25.9(\mathrm{C}-14)$, *these data were not obtained by $2 \mathrm{D}{ }^{1} \mathrm{H} /{ }^{13} \mathrm{C}$ correlated spectroscopy (COSY) spectrum; assignments exchangeable; HRMS $m / z$, calcd. for $\mathrm{C}_{19} \mathrm{H}_{18} \mathrm{O}_{3}[\mathrm{M}+\mathrm{H}]^{+}$: 295.1455, found: 295.1334 .

1-Oxo-1H-isochromen-6-yl (E)-3-(4-methoxyphenyl)acrylate (10)

Following the general procedure, and using 6-hydroxy$1 \mathrm{H}$-isocromen-1-one ( $53.5 \mathrm{mg}, 0.33 \mathrm{mmol}$ ), compound $\mathbf{1 0}$ was obtained as amorphous white solid in $89.9 \%$ yield; $\mathrm{mp}>200{ }^{\circ} \mathrm{C}$; IR (KBr) v / $\mathrm{cm}^{-1} 3072,2933,1735,1622$, $1602,1514,1255,1139,1120,995,821 ;{ }^{1} \mathrm{H}$ NMR $\left(300 \mathrm{MHz}, \mathrm{CDCl}_{3}\right) \delta 7.87$ (d, 1H, J 15.9 Hz, H-7), 7.72 (d, $1 \mathrm{H}, J 9.6 \mathrm{~Hz}, \mathrm{H}-14), 7.56$ (d, 2H, J 8.7 Hz, H-3, H-5), 7.52 (d, 1H, J 8.5 Hz, H-17), 7.21 (d, 1H, J 2.0 Hz, H-11), 7.14 (dd, 1H, J 8.4, $2.1 \mathrm{~Hz}, \mathrm{H}-18), 6.96$ (d, 2H, J $8.7 \mathrm{~Hz}, \mathrm{H}-2$, H-6), 6.49 (d, 1H, J 15.9 Hz, H-8), 6.41 (d, 1H, J 9.6 Hz, $\mathrm{H}-13), 3.92$ (s, 3H, $\left.\mathrm{CH}_{3} \mathrm{O}-1 \mathrm{a}\right) ;{ }^{13} \mathrm{C} \mathrm{NMR}\left(75 \mathrm{MHz}, \mathrm{CDCl}_{3}\right.$ ) $\delta 165.2$ (C-9), 162.2 (C-15), 160.6 (C-1), 154.9 (C-10), 153.7 (C-12), 147.6 (C-7), 143.1 (C-14), 130.4 (C-3, C-5), 128.7 (C-17), 126.8 (C-4), 118.7 (C-18), 116.7 (C-16), 116.1 (C-8), 114.7 (C-2, C-6), 113.9 (C-11), 110.7 (C-13), $55.6\left(\mathrm{CH}_{3} \mathrm{O}-1 \mathrm{a}\right)$; HRMS $m / z$, calcd. for $\mathrm{C}_{19} \mathrm{H}_{14} \mathrm{O}_{5}[\mathrm{M}+\mathrm{H}]^{+}$: 323.0890, found: 323.0919 .

(S)-(4-(Prop-1-en-2-yl)cyclohex-1-en-1-yl)methyl (E)-3-(4methoxyphenyl)acrylate (11)

Following the general procedure, and using (S)-(-)-perillyl alcohol (48 $\mu \mathrm{L}, 0.33 \mathrm{mmol})$, compound 11 was obtained as yellow liquid in $81.3 \%$ yield; IR $(\mathrm{KBr})$ $v / \mathrm{cm}^{-1} 3074,2926,2827,1710,1635,1606,1438,1253$, 1159, 827, 518; ${ }^{1} \mathrm{H}$ NMR (300 MHz, $\left.\mathrm{CDCl}_{3}\right) \delta 7.67$ (d, $1 \mathrm{H}, J 16.0 \mathrm{~Hz}, \mathrm{H}-7), 7.49$ (d, 2H, J 8.7 Hz, H-2, H-6), 6.91 (d, 2H, J 8.7 Hz, H-3, H-5), 6.35 (d, 1H, J 16.0 Hz, H-8), 
5.82 (brs, $1 \mathrm{H}, \mathrm{H}-12$ ), 4.74 (brs, $2 \mathrm{H}, 2 \mathrm{H}-18$ ), 4.60 (s, $2 \mathrm{H}$, $2 \mathrm{H}-10$ ), 3.85 (s, 3H, $\left.\mathrm{CH}_{3} \mathrm{O}-1 \mathrm{a}\right), 2.18$ (m, 4H, H-16, H-16', H-14, H-13), 2.03 (m, 1H, H-13'), 1.87 (m, 1H, H-15), 1.75 (s, 3H, 3H-19), 1.53 (m, 1H, H-15'); ${ }^{13} \mathrm{C}$ NMR (75 MHz, $\left.\mathrm{CDCl}_{3}\right) \delta 167.4$ (C-9), $161.6(\mathrm{C}-4), 149.8$ (C-17), 144.7 (C-7), 133.0 (C-11), 129.9 (C-2, C-6), 127.4 (C-1), 125.8 (C-12), 115.8 (C-8), 114.5 (C-3, C-5), 108.9 (C-18), 68.5 (C-10), $55.6\left(\mathrm{CH}_{3} \mathrm{O}-1 \mathrm{a}\right), 41.1$ (C-14), 30.7 (C-16), 27.5 (C-13), 26.7 (C-15), 20.9 (C-19); HRMS $\mathrm{m} / \mathrm{z}$, calcd. for $\mathrm{C}_{20} \mathrm{H}_{24} \mathrm{O}_{3}[\mathrm{M}+\mathrm{H}]^{+}:$313.1804, found: 313.1790 .

\section{3-Methoxybenzyl (E)-3-(4-methoxyphenyl)acrylate (12)}

Following the general procedure, and using 3-methoxybenzyl alcohol ( $46 \mu \mathrm{L}, 0.33 \mathrm{mmol})$, compound 12 was obtained as yellow liquid in $88.7 \%$ yield; IR $(\mathrm{KBr})$ $v / \mathrm{cm}^{-1}$ 2935, 2835, 1710, 1631, 1602, 1512, 1460, 1253, $1157,1029,829 ;{ }^{1} \mathrm{H} \mathrm{NMR}\left(300 \mathrm{MHz}, \mathrm{CDCl}_{3}\right) \delta 7.70(\mathrm{~d}, 1 \mathrm{H}$, $J 16.0 \mathrm{~Hz}, \mathrm{H}-7), 7.49$ (d, 2H, J 8.7 Hz, H-2, H-6), 7.29 (t, $1 \mathrm{H}, J 9.0 \mathrm{~Hz}, \mathrm{H}-15$ ), 7.02 (brs, 1H, H-12), 6.97-6.90 (m, $2 \mathrm{H}$, H-16, H-14), 6.92 (d, 2H, J 8.7 Hz, H-3, H-5), 6.37 (d, 1H, $J 16.0 \mathrm{~Hz}, \mathrm{H}-8), 5.23$ (s, $2 \mathrm{H}, 2 \mathrm{H}-10), 3.84$ (s, 3H, $\mathrm{CH}_{3} \mathrm{O}-1 \mathrm{a}$ ), $3.84\left(\mathrm{~s}, 3 \mathrm{H}, \mathrm{CH}_{3} \mathrm{O}-17\right) ;{ }^{13} \mathrm{C}$ NMR $\left(75 \mathrm{MHz}, \mathrm{CDCl}_{3}\right)$ $\delta 167.3$ (C-9), 161.6 (C-4), 159.9 (C-13), 145.1 (C-7), 137.9 (C-11), 129.9 (C-2, C-6)*, $129.8(\mathrm{C}-15)^{*}, 127.3$ (C-1), 120.6 (C-16), 115.5 (C-8), 114.5 (C-3, C-5), 113.9 $(\mathrm{C}-14)^{* *}, 113.8(\mathrm{C}-12)^{* *}, 66.2(\mathrm{C}-10), 55.6\left(\mathrm{CH}_{3} \mathrm{O}-1 \mathrm{a}\right)$, $55.5\left(\mathrm{CH}_{3} \mathrm{O}-17\right)$, *exchageable assignments, **exchageable assignments; HRMS $m / z$, calcd. for $\mathrm{C}_{18} \mathrm{H}_{18} \mathrm{O}_{4}[\mathrm{M}+\mathrm{H}]^{+}$: 299.1316, found: 299.1285 .

\section{2-Isopropyl-5-methylphenyl (E)-3-(4-methoxyphenyl)acrylate} (13)

Following the general procedure, and using thymol (49.6 mg, $0.33 \mathrm{mmol}$ ), compound $\mathbf{1 3}$ was obtained as white solid $79.1 \%$ yield; $\mathrm{mp} 82-84{ }^{\circ} \mathrm{C}$; IR $(\mathrm{KBr}) v / \mathrm{cm}^{-1} 2955$, 2927, 2852, 2117, 1722, 1635, 1600, 1512, 1456, 1259, $1139,833,526 ;{ }^{1} \mathrm{H}$ NMR $\left(300 \mathrm{MHz}, \mathrm{CDCl}_{3}\right) \delta 7.85(\mathrm{~d}, 1 \mathrm{H}$, $J 15.9 \mathrm{~Hz}, \mathrm{H}-7), 7.56$ (d, 2H, J 8.7 Hz, H-2, H-6), 7.26 (d, $1 \mathrm{H}, J 7.9 \mathrm{~Hz}, \mathrm{H}-12$ ), 7.05 (brd, $1 \mathrm{H}, J 7.7 \mathrm{~Hz}, \mathrm{H}-13$ ), 6.96 (d, 2H, J 8.6 Hz, H-3, H-5), 6.90 (brs, 1H, H-15), 6.54 (d, $1 \mathrm{H}, J 15.9 \mathrm{~Hz}, \mathrm{H}-8), 3.87$ (s, 3H, $\left.\mathrm{CH}_{3} \mathrm{O}-1 \mathrm{a}\right), 3.05$ (q, $1 \mathrm{H}$, $J 6.0 \mathrm{~Hz}, \mathrm{H}-16), 2.34$ (s, 3H, 3H-19), 1.22 (d, 6H, J6.0 Hz, $3 \mathrm{H}-17,3 \mathrm{H}-18) ;{ }^{13} \mathrm{C}$ NMR $\left(75 \mathrm{MHz}, \mathrm{CDCl}_{3}\right) \delta 166.2(\mathrm{C}-9)$, 161.9 (C-4), 148.2 (C-11), 146.3 (C-7), 137.4 (C-14), 136.7 (C-11), 130.2 (C-2, C-6), 126.7 (C-1), 127.2 (C-12), 126.7 (C-13), 123.1 (C-15), 114.9 (C-8), 114.6 (C-3, C-5), 55.6 $\left(\mathrm{CH}_{3} \mathrm{O}-1 \mathrm{a}\right), 27.3$ (C-16), 23.2 (C-17, C-18), 21.0 (C-19); HRMS $m / z$, calcd. for $\mathrm{C}_{20} \mathrm{H}_{22} \mathrm{O}_{3}[\mathrm{M}+\mathrm{H}]^{+}: 311.1650$, found: 311.1647. Data are in agreement with those previously reported. ${ }^{20}$
5-Isopropyl-2-methylphenyl (E)-3-(4-methoxyphenyl) acrylate (14)

Following the general procedure, and using carvacrol (48 $\mu \mathrm{L}, 0.33 \mathrm{mmol}$ ), compound $\mathbf{1 4}$ was obtained as amorphous white solid in $86 \%$ yield; mp $75-77{ }^{\circ} \mathrm{C}$; IR (KBr) v / $\mathrm{cm}^{-1} 3051,2958,2927,2866,1722,1633$, 1600, 1510, 1460, 1313, 1255, 1138, 825, 528; ${ }^{1} \mathrm{H}$ NMR $\left(300 \mathrm{MHz}, \mathrm{CDCl}_{3}\right) \delta 7.86(\mathrm{~d}, 1 \mathrm{H}, J 15.9 \mathrm{~Hz}, \mathrm{H}-7), 7.56$ (d, 2H, J 8.7 Hz, H-2, H-6), 7.18 (d, 1H, J 7.8 Hz, H-12), 7.06 (brd, 1H, H-13), 6.97 (brs, 1H, H-15), 6.95 (d, 2H, $J 8.7 \mathrm{~Hz}, \mathrm{H}-3, \mathrm{H}-5), 6.55$ (d, 1H, J $15.9 \mathrm{~Hz}, \mathrm{H}-8), 3.87$ (s, $3 \mathrm{H}, \mathrm{CH}_{3} \mathrm{O}-1 \mathrm{a}$ ), 2.91 (q, $1 \mathrm{H}, J 6.0 \mathrm{~Hz}, \mathrm{H}-16$ ), 2.19 (s, 3H, $3 \mathrm{H}-19), 1.26$ (d, 6H, J 6.0 Hz, 3H-17, 3H-18); ${ }^{13} \mathrm{C}$ NMR (75 MHz, $\left.\mathrm{CDCl}_{3}\right) \delta 165.7$ (C-9), 161.9 (C-4), $149.6(\mathrm{C}-10)$, 148.2 (C-14), 146.3 (C-7), 131.1 (C-12), 130.2 (C-2, C-6), $127.6(\mathrm{C}-11)^{*}, 127.2(\mathrm{C}-1)^{*}, 124.2(\mathrm{C}-13), 120.1(\mathrm{C}-15)$, $114.8(\mathrm{C}-8), 114.6(\mathrm{C}-3, \mathrm{C}-5), 55.6\left(\mathrm{CH}_{3} \mathrm{O}-1 \mathrm{a}\right), 33.7(\mathrm{C}-16)$, 24.1 (C-17, C-18), 16.1 (C-19), *exchageable assignments; HRMS $m / z$, calcd. for $\mathrm{C}_{20} \mathrm{H}_{22} \mathrm{O}_{3}[\mathrm{M}+\mathrm{H}]^{+}: 311.1650$, found: 311.1647 .

4-Formyl-2-methoxyphenyl (E)-3-(4-methoxyphenyl)acrylate (15)

Following the general procedure, and using vanillin (50.2 $\mathrm{mg}, 0.33 \mathrm{mmol}$ ), compound $\mathbf{1 5}$ was obtained as yellow crystalline solid in $93.8 \%$ yield; $\mathrm{mp} 87-89^{\circ} \mathrm{C}$; IR $(\mathrm{KBr}) \vee / \mathrm{cm}^{-1} 2935,2845,1722,1703,1624,1598$, 1506, 1257, 1134, 1026, 833, 526; ' $\mathrm{H}$ NMR (300 MHz, $\left.\mathrm{CDCl}_{3}\right) \delta 9.97(\mathrm{~s}, 1 \mathrm{H}, \mathrm{H}-16), 7.86(\mathrm{~d}, 1 \mathrm{H}, J 15.9 \mathrm{~Hz}, \mathrm{H}-7)$, 7.56 (d, 2H, J 8.5 Hz, H-2, H-6), 7.53 (brs, 1H, H-12), 7.51 (brd, $1 \mathrm{H}, J 8.5 \mathrm{~Hz}, \mathrm{H}-14), 7.31$ (d, 1H, J 8.5 Hz, H-15), $6.95(\mathrm{~d}, 2 \mathrm{H}, J 8.5 \mathrm{~Hz}, \mathrm{H}-3, \mathrm{H}-5), 6.53(\mathrm{~d}, 1 \mathrm{H}, J 15.9 \mathrm{~Hz}$, $\mathrm{H}-8$ ), 3.92 (s, 3H, $\mathrm{CH}_{3} \mathrm{O}-1 \mathrm{a}$ ), 3.86 (s, 3H, $\mathrm{CH}_{3} \mathrm{O}-17$ ); ${ }^{13} \mathrm{C}$ NMR (75 MHz, $\left.\mathrm{CDCl}_{3}\right) \delta 191.1$ (C-16), 164.6 (C-9), 161.9 (C-4), 152.2 (C-10), 147.1 (C-7), 145.2 (C-11), 135.2 (C-13), 130.2 (C-2, C-6), 126.8 (C-1), 124.8 (C-14), 123.6 (C-15), 114.5 (C-3, C-5), 113.7 (C-8), 110.9 (C-12), $56.1\left(\mathrm{CH}_{3} \mathrm{O}-1 \mathrm{a}\right), 55.4\left(\mathrm{CH}_{3} \mathrm{O}-17\right)$; HRMS $\mathrm{m} / z$, calcd. for $\mathrm{C}_{18} \mathrm{H}_{16} \mathrm{O}_{5}[\mathrm{M}+\mathrm{H}]^{+}:$313.1085, found: 313.1076 .

rac-(2R,5R)-2-Methyl-5-(prop-1-en-2-yl)cyclohexyl $(E)$-3-(4-methoxyphenyl)acrylate (16)

Following the general procedure, and using rac-dihydrocarveol $(55 \mu \mathrm{L}, 0.33 \mathrm{mmol})$, compound $\mathbf{1 6}$ was obtained as translucent liquid $86.5 \%$ yield; IR (KBr) $v / \mathrm{cm}^{-1} 2931,2858,1707,1633,1604,1512,1253,1168$, 829; ${ }^{1} \mathrm{H}$ NMR $\left(300 \mathrm{MHz}, \mathrm{CDCl}_{3}\right) \delta 7.65(\mathrm{~d}, 1 \mathrm{H}, J 15.9 \mathrm{~Hz}$, H-7), 7.49 (d, 2H, J $8.5 \mathrm{~Hz}, \mathrm{H}-2, \mathrm{H}-6), 6.91(\mathrm{~d}, 2 \mathrm{H}$, $J 8.5 \mathrm{~Hz}, \mathrm{H}-3, \mathrm{H}-5), 6.32$ (d, 1H, J $15.9 \mathrm{~Hz}, \mathrm{H}-8), 4.70$ (s, 2H, 2H-17), 4.62 (td, 1H, J 10.7, $3.9 \mathrm{~Hz}, \mathrm{H}-10), 3.84$ (s, $\left.3 \mathrm{H}, \mathrm{CH}_{3}-1 \mathrm{a}\right)$ 2.24-2.02 (m, 2H, H-14, H-15), 1.85 (d, 1H, 
$J 10.1 \mathrm{~Hz}, \mathrm{H}-15$ ') 1.73 (s, 3H, 3H-18), 1.70-1.60 (m, 2H, H-11, H-13), 1.36-1.15 (m, 2H, 2H-12, H-13'), 0.96 (d, 3H, $J 6.5 \mathrm{~Hz}, 3 \mathrm{H}-19) ;{ }^{13} \mathrm{C} \mathrm{NMR}\left(75 \mathrm{MHz}, \mathrm{CDCl}_{3}\right) \delta 167.1(\mathrm{C}-9)$, 161.3 (C-4), 149.0 (C-17), 144.2 (C-7), 129.7 (C-2, C-6), 127.3 (C-1), 116.1 (C-8), 114.3 (C-3, C-5), 108.8 (C-17), 78.1 (C-10), $55.39\left(\mathrm{CH}_{3} \mathrm{O}-1 \mathrm{a}\right), 43.7(\mathrm{C}-11), 37.4(\mathrm{C}-14)^{*}$, 37.0 (C-15)*, 33.2 (C-12), 30.9 (C-13), 20.9 (C-19), 18.3 (C-18); HRMS $m / z$, calcd. for $\mathrm{C}_{20} \mathrm{H}_{26} \mathrm{O}_{3}[\mathrm{M}+\mathrm{H}]^{+}: 315.1962$, found: 315.1960 .

\section{5-Isopropyl-2-methylphenyl (E)-3-(3-hydroxy-4- methoxyphenyl)acrylate (17)}

Following the general procedure, and using carvacrol $(64.0 \mu \mathrm{L}, 0.44 \mathrm{mmol})$, compound $\mathbf{1 7}$ was obtained as yellow pasty liquid $18.6 \%$ yield; IR $(\mathrm{KBr}) \mathrm{v} / \mathrm{cm}^{-1}$ 3425, 2960, 2922, 2852, 1720, 1631, 1591, 1512, 1269 , 1232, 1134; ${ }^{1} \mathrm{H}$ NMR $\left(300 \mathrm{MHz}, \mathrm{CDCl}_{3}\right) \delta 7.83(\mathrm{~d}, 1 \mathrm{H}$, $J 15.9 \mathrm{~Hz}, \mathrm{H}-7), 7.17$ (d, 1H, J 8.1 Hz, H-12), 7.15 (brd, 1H, H-6), 7.12 (s, 1H, H-2), 7.03 (brd, 1H, H-13), 6.98 (d, 1H, J $8.1 \mathrm{~Hz}, \mathrm{H}-5), 6.95$ (s, 1H, H-15), 6.53 (d, 1H, $J 15.9 \mathrm{~Hz}, \mathrm{H}-8$ ), 3.97 (s, $\left.3 \mathrm{H}, \mathrm{CH}_{3} \mathrm{O}-1 \mathrm{a}\right), 2.93-2.81$ (sex, $1 \mathrm{H}, J 6.0 \mathrm{~Hz}, \mathrm{H}-16), 2.19$ (s, 3H, 3H-19), 1.26 (d, 6H, $J 6.1 \mathrm{~Hz}, 3 \mathrm{H}-17,3 \mathrm{H}-18) ;{ }^{13} \mathrm{C} \mathrm{NMR}\left(75 \mathrm{MHz}, \mathrm{CDCl}_{3}\right) \delta$ 166.8 (C-9), 149.0 (C-10), 148.5 (C-4)*, 148.3 (C-14)*, 147.1 (C-3), 146.7 (C-7), 131.1 (C-12), 127.6 (C-11), 126.9 (C-1), 124.6 (C-13), 123.6 (C-6), 120.1 (C-15), 115.0 (C-8), 114.8 (C-5), $113.2(\mathrm{C}-2), 56.2\left(\mathrm{CH}_{3} \mathrm{O}-1 \mathrm{a}\right)$, 33.8 (C-16), 29.9 (C-18), 24.1 (C-17), 16.1 (C-19), *exchangeable assignments; HRMS $\mathrm{m} / \mathrm{z}$, calcd. for $\mathrm{C}_{20} \mathrm{H}_{26} \mathrm{O}_{3}[\mathrm{M}+\mathrm{H}]^{+}:$327.1597, found: 327.1596 .

\section{2-Isopropyl-5-methylphenyl (E)-3-(3-hydroxy-4- methoxyphenyl)acrylate (18)}

Following the general procedure, and using thymol (66.0 $\mathrm{mg}, 0.44 \mathrm{mmol}$ ), compound $\mathbf{1 8}$ was obtained as yellow pasty liquid $15.5 \%$ yield; IR $(\mathrm{KBr}) \vee / \mathrm{cm}^{-1} 3398$, 2935, 2843, 1718, 1629, 1591, 1514, 1271, 1230, 1138, 813; ${ }^{1} \mathrm{H}$ NMR (300 MHz, $\left.\mathrm{CDCl}_{3}\right) \delta 7.82(\mathrm{~d}, 1 \mathrm{H}, J 15.9 \mathrm{~Hz}$, H-7), 7.23 (d, 1H, J 7.9 Hz, H-12), 7.15 (brd, $1 \mathrm{H}, J 8.2 \mathrm{~Hz}$, H-6), 7.12 (brs, 1H, H-2), 7.06 (brd, 1H, J $8.2 \mathrm{~Hz}, \mathrm{H}-13$ ), 6.97 (d, 1H, J 8.1 Hz, H-5), 6.89 (brs, 1H, H-15), 6.52 (d, $1 \mathrm{H}, J 15.9 \mathrm{~Hz}, \mathrm{H}-8$ ), 3.96 (s, 3H, $\left.\mathrm{CH}_{3} \mathrm{O}-1 \mathrm{a}\right), 3.12-2.98$ (epi, 1H, J 6.1 Hz, H-16), 2.34 (s, 3H, 3H-19), 1.22 (d, $6 \mathrm{H}, J 6.0 \mathrm{~Hz}, 3 \mathrm{H}-17,3 \mathrm{H}-18)$; ${ }^{13} \mathrm{C}$ NMR $\left(75 \mathrm{MHz}, \mathrm{CDCl}_{3}\right)$ $\delta 166.2(\mathrm{C}-9), 148.5(\mathrm{C}-4)^{*}, 148.0(\mathrm{C}-10)^{*}, 147.1(\mathrm{C}-3)$, 146.7 (C-7), 137.4 (C-14), 136.7 (C-11), 127.2 (C-12), 126.9 (C-1), 126.6 (C-13), 123.6 (C-6)**, 123.1 (C-15)*, 115.0 (C-8), 114.8 (C-5), 109.7 (C-2), $56.2\left(\mathrm{CH}_{3} \mathrm{O}-1 \mathrm{a}\right)$, 27.3 (C-16), 23.3 (C-17, C-18), 21.0 (C-19), *exchangeable assignments, **exchangeable assignments; HRMS $\mathrm{m} / \mathrm{z}$, calcd. for $\mathrm{C}_{20} \mathrm{H}_{26} \mathrm{O}_{3}[\mathrm{M}+\mathrm{H}]^{+}:$327.1576, found: 327.1596 .
The structure of compound 6 is reported in the literature, ${ }^{24}$ but it does not report spectroscopic data.

\section{Cytotoxicity}

The cytotoxic activity of cinnamates was evaluated in SNB-19 (astrocytoma), HCT-116 (colon carcinoma), PC3 (prostate carcinoma), HL60 (promyelocytic leukemia), which were obtained from the National Cancer Institute (Washington, USA). All cells were cultured in Roswell Park Memorial Institute (RPMI) 1640, except for L929, which was cultivated in Dulbecco's Modified Eagle Medium (DMEM), obtained from the Rio de Janeiro Cell Bank (BCRJ) (Rio de Janeiro, Brazil). All cell culture experiments were performed at $37{ }^{\circ} \mathrm{C}$. Cells were supplemented with $10 \%$ fetal calf serum and $1 \%$ of antibiotics, in a $5 \% \mathrm{CO}_{2}$ humidified atmosphere. The L929 cell line was used to evaluate the selectivity of the extracts and these assays, the anticancer drug doxorubicin was used as positive control.

Cytotoxicity assays were carried out essentially according the MTT colorimetric method [3-(4,5-dimethyl2-thiazolyl)-2,5-diphenyl-2H-tetrazolium]. ${ }^{25-27}$ The compounds were tested at $25 \mu \mathrm{g} \mathrm{mL}^{-1}$ in four lines tumor cells for initial screening; the half maximal inhibitory concentration $\left(\mathrm{IC}_{50}\right)$ was determined for samples that showed positive results (growth inhibition $>70 \%$ ) in at least one cell line. The cells were plated in 96-well plates at the following concentrations: HCT-116/L929: $0.7 \times 10^{5}$ cells mL ${ }^{-1}$; SNB-19/PC3: $0.1 \times 10^{6}$ cells mL ${ }^{-1}$; HL60: $0.3 \times 10^{6}$ cells $\mathrm{mL}^{-1}$. The cells were treated with the substances for $72 \mathrm{~h}$. At the end of the treatment, the plates were centrifuged and the supernatant removed. Then, $100 \mu \mathrm{L}$ of MTT solution $\left(0.5 \mu \mathrm{g} \mathrm{mL}^{-1}\right)$ were added and incubated for $3 \mathrm{~h}$. After incubation, the MTT solution was removed, and the precipitated formazan was dissolved with $100 \mu \mathrm{L}$ of dimethyl sulfoxide (DMSO). The absorbances were read using a plate spectrophotometer (Multimode Detector, DTX 880, Beckman Coulter) provided by Analytical Instruments (Golden Valley, USA) at $595 \mathrm{~nm}$.

\section{Statistical analysis}

All experiments were performed in duplicate and repeated three times. For samples that showed $>70 \%$ inhibitory, activity the selectivity index (SI) was calculated. The calculation of this index corresponds to the division between the $\mathrm{IC}_{50}$ value of each test compound in the nontumor cell line $\mathrm{L} 929$ and the $\mathrm{IC}_{50}$ value of each compound in the tumor cell line $\left(\mathrm{SI}=\right.$ neoplastic cells $\left.\mathrm{IC}_{50} \mathrm{~L} 929 / \mathrm{IC}_{50}\right) .{ }^{28}$ The results obtained were analyzed using the GraphPad 
Prism 5.01 software and expressed as mean \pm standard deviation. ${ }^{29} \mathrm{IC}_{50}$ values were obtained by interpolation from non-linear regression analysis with a $95 \%$ confidence level. $\mathrm{IC}_{50}$ was defined as the concentration sufficient to obtain $50 \%$ of the maximum inhibitory effect on cell viability.

\section{Results and Discussion}

As previously described, derivatives of phenylpropanoid acids exhibit various biological activities, including anticancer. Considering these important aspects, in the present study eighteen esters derived from cinnamic (1a), $p$-methoxycinnamic (2a) and ferulic (3a) acids were synthesized. It is interesting to note that the relationship between molecular structure and pharmacological activity is studied according to several parameters, including electronic, steric and stereochemical. The esters derived here synthesized contain the cinnamoyl residue, cited as a biologically active molecular fragment, while the alcohol/ phenol part gives the structure a steric and/or stereogenic dimension.

Structural modifications from cinnamic (1a), $p$-methoxycinnamic (2a) and ferulic (3a) acids carried out on the carboxyl group, were represented in Schemes 1, 2 and 3, respectively. The esters 1-8 (Scheme 1) and 9-16 (Scheme 2) were synthesized according to the esterification of Steglich, ${ }^{19}$ which is a variation of an esterfication with $N, N^{\prime}$-dicyclohexylcarbodiimide (DCC) as a coupling reagent and 4-( $N, N^{\prime}$-dimethylamino)pyridine (DMAP) as a catalyst. In turn, 3-methoxy-4-hydroxy-cinnamic acid after acetylation with acetic anhydride in the presence of triethylamine $\left(\mathrm{Et}_{3} \mathrm{~N}\right)$ and DMAP gave the corresponding acetate (3b), which was then esterified via Sterlich to give 17 and $\mathbf{1 8}$ (Scheme 3). For all esters, yields were in the range 76.6-95\%, except 17 (18.6\%) and $\mathbf{1 8}$ (15.5\%).

Among all the synthesized cinnamates, compounds $\mathbf{1}$, $2,3,8,9,10,11,12,14,15$ and 16, no synthesis and biological studies were reported, being unpublished. However, compounds 4, 5, 6, 7, 13, 17 and 18 have been reported and exhibited antimicrobial activity, ${ }^{1720-24}$ but there are no cytotoxicity test studies on any cell line. Thus, the cytotoxic activity of all esters derived from cinnamic acids against four human cancer cell lines was evaluated: SNB-19 (astrocytoma), HCT-116 (colon carcinoma, human), PC3 (prostate) and HL60 (promyelocytic leukemia). We use the MTT colorimetric method developed by Mosmann. ${ }^{25}$

All the compounds have been characterized by IR, HRMS and NMR $\left({ }^{1} \mathrm{H}\right.$ and $\left.{ }^{13} \mathrm{C}\right)$ spectral data. Some spectral data were characteristic and common to all, as expected. Thus, for all esters, the spectra: IR showed absorption bands in the range of $1726-1707 \mathrm{~cm}^{-1}$ due to stretches of carbonyl groups, as expected for stretches of conjugated ester carbonyl groups; ${ }^{1} \mathrm{H}$ NMR showed a characteristic spin system consisting of trans olefinic protons arranged in a polarized carbon-carbon double bond $\left(\delta_{\mathrm{H}} 7.19\right.$ and $7.86, J 16 \mathrm{~Hz}) ;{ }^{13} \mathrm{C}$ NMR, signals around $\delta_{\mathrm{C}} 166.00$, also consistent with conjugated esters carbonyl carbons. The ${ }^{1} \mathrm{H}$ and ${ }^{13} \mathrm{C}$ NMR assignments were performed taking into account aspects such as chemical shifts, multiplicity and coupling constants, displayed by the signals in the respective spectra. In addition, by comparison with data recorded in the literature for compounds of the same nature. ${ }^{17,20,23,30}$ The ${ }^{1} \mathrm{H}$ and ${ }^{13} \mathrm{C}$ NMR data of these compounds are given in the Experimental section.

Initially, the esters were screened using a program from the National Cancer Institute (NCI) ${ }^{31}$ which easily allows a qualitative or semi-quantitative analysis to determine cytotoxicity. ${ }^{25}$ An intensity scale was used to assess the cytotoxic potential of products, according to the following results: $1-50 \%$ (low or medium), $50-75 \%$ (moderate) and $75-100 \%$ (high). ${ }^{32,33}$ According to this program, of the eighteen synthesized compounds, eleven exhibited cytotoxic potential with cell growth inhibition above 50\% and six with $75-100 \%$ inhibition (high activity). Thus, based on the initial screening, compounds $6,8,12,14$, 15 and 18 showed promising cytotoxicity, with $>70 \%$ inhibition of cells proliferation in at least one of the lines tested. Only those with an inhibition percentage above $70 \%$ were evaluated for the mean inhibitory concentration $\left(\mathrm{IC}_{50}=\right.$ concentration causing a $50 \%$ inhibition) (Table 1). For the other compounds, the results were not satisfactory, with the value $<70 \%$ inhibition. The results were summarized in the cell inhibition (Figures S1-S4) which can be found in the Supplementary Information section.

As summarized in Table 1, the six compounds demonstrated a general (non specific) cytotoxic response. For example, the intensity of the response demonstrated by compound $6\left(\mathrm{IC}_{50} 23.2 \mu \mathrm{M}\right)$ was similar to that demonstrated by $8\left(\mathrm{IC}_{50} 25.2 \mu \mathrm{M}\right)$, however, on different cell lines, that is, HCT-116 and HL60, respectively. In the 2a-compounds series, $12\left(\mathrm{IC}_{50} 16.2 \mu \mathrm{M}\right)$ demonstrated a cytotoxic response greater than $\mathbf{1 4}\left(\mathrm{IC}_{50} 29.3 \mu \mathrm{M}\right)$ against the HCT-116 strain. The highest intensity of response was shown by compound 18 against HTC- 116 cells with an $\mathrm{IC}_{50}$ of $15.4 \mu \mathrm{M}$. All compounds showed higher $\mathrm{IC}_{50}$ values with respect to doxorubicin in all strains. Regarding the cytotoxicity of the compounds against the non-tumor cell line (L929), the samples showed $\mathrm{IC}_{50}>77 \mu \mathrm{M}$, demonstrating that the samples have low cytotoxicity against non-tumor cell lines. With the exception of the compound $\mathbf{6}$, which showed inhibitory activity against L929 cells with $\mathrm{IC}_{50}$ values of $32.5 \mu \mathrm{M}$. 
Table 1. $\mathrm{IC}_{50}$ values of compounds $\mathbf{6}, \mathbf{8}, \mathbf{1 2}, \mathbf{1 4}, \mathbf{1 5}$ and $\mathbf{1 8}$ in tumor and non-tumor cell lines with a $95 \%$ confidence interval

\begin{tabular}{|c|c|c|c|c|c|}
\hline \multirow{2}{*}{ Compound } & \multicolumn{5}{|c|}{$\mathrm{IC}_{50} / \mu \mathrm{M}$} \\
\hline & HL60 & SNB-19 & HCT-116 & PC3 & L929 \\
\hline 6 & $>89^{a}$ & $38.3 \pm 11.3$ & $23.2 \pm 3.5$ & $49.0 \pm 5.9$ & $32.5 \pm 21.4$ \\
\hline 8 & $25.2 \pm 3.0$ & $>88^{\mathrm{a}}$ & $>88^{\mathrm{a}}$ & $>88^{\mathrm{a}}$ & $>88^{\mathrm{a}}$ \\
\hline 12 & $>84^{\mathrm{a}}$ & $42.1 \pm 14.2$ & $16.2 \pm 6.3$ & $41.9 \pm 7.9$ & $>84^{\mathrm{a}}$ \\
\hline 14 & $>80^{\mathrm{a}}$ & $>80^{\mathrm{a}}$ & $29.3 \pm 7.5$ & $68.1 \pm 3.6$ & $>80^{\mathrm{a}}$ \\
\hline 15 & $79.8 \pm 16.4$ & $>80^{\mathrm{a}}$ & $>80^{\mathrm{a}}$ & $>80^{\mathrm{a}}$ & $>80^{\mathrm{a}}$ \\
\hline 18 & $>77^{\mathrm{a}}$ & $>77$ & $15.38 \pm 1.6$ & $67.4 \pm 18.1$ & $>77^{\mathrm{a}}$ \\
\hline $\mathrm{DOX}^{\mathrm{b}}$ & $0.01 \pm 0.009$ & $3.8 \pm 0.6$ & $0.35 \pm 0.09$ & $1.4 \pm 0.3$ & $3.2 \pm 0.27$ \\
\hline
\end{tabular}

anhibition of proliferation did not exceed $50 \%$ at the highest concentration tested; ${ }^{b}$ doxorubicin was used as a positive control. IC C $_{50}$ : half maximal inhibitory concentration; HL60: promyelocytic leukemia; SNB-19: astrocytoma, HCT-116: colon carcinoma, human; PC3: prostate; L929: cell murine fibroblast.

The comparison between the activity in relation to neoplastic cell lines and normal cells (L929) was made to calculate the selectivity index (SI), as an indication of the potential of using the compounds for future clinical tests. Ideally, the drug should only kill patient cancer cells without significantly affecting healthy cells. SI is considered significant when it has a value greater than or equal to 2.0 , that is, this value means that the compound has activity twice in the lineages of neoplastic cells than in normal cells. ${ }^{28}$ For the compounds $6,8,12,14,15$ and 18 the SI was calculated, which can be seen in Table 2.

Table 2. Values of the selectivity index (SI) of the tested compounds. SI was calculated for each compound using the formula: $\mathrm{SI}=\mathrm{IC}_{50}$ normal cells/IC 50 of the respective cancer cells

\begin{tabular}{lcccc}
\hline \multirow{2}{*}{ Compound } & \multicolumn{4}{c}{ SI } \\
\cline { 2 - 5 } & HL60 & SNB-19 & HCT-116 & PC3 \\
\hline $\mathbf{6}$ & - & 0.84 & 1.4 & 0.7 \\
$\mathbf{8}$ & $-^{\mathrm{a}}$ & - & - & - \\
$\mathbf{1 2}$ & - & $-{ }^{\mathrm{a}}$ & $-^{\mathrm{a}}$ & $-^{\mathrm{a}}$ \\
$\mathbf{1 4}$ & - & - & $-^{\mathrm{a}}$ & $-^{\mathrm{a}}$ \\
$\mathbf{1 5}$ & $-^{\mathrm{a}}$ & - & - & - \\
$\mathbf{1 8}$ & - & - & $-^{\mathrm{a}}$ & $-^{\mathrm{a}}$ \\
\hline
\end{tabular}

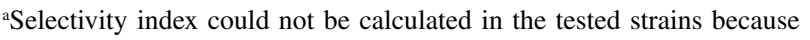
the substances did not present $\mathrm{IC}_{50}$ calculable for the non-tumoral strain $\left(\mathrm{IC}_{50}>77 \mu \mathrm{M}\right) . \mathrm{IC}_{50}$ : half maximal inhibitory concentration; HL60: promyelocytic leukemia; SNB-19: astrocytoma, HCT-116: colon carcinoma, human; PC3: prostate.

For all strains tested, SI values were $>2$ showing that the compounds are selective between neoplastic and normal cells. Combining SI with antiproliferative activity, the substances become candidates for drugs for future studies. However, a notable exception was the compound $\mathbf{6}$, with SI values of $0.84,1.4$ and 0.7 for the SNB-19, HCT-116 and PC3 strains, mutually. It is important to note that the compound $\mathbf{6}$ showed a high toxicity, preferentially inhibiting normal cells than neoplastic cells SNB-19 and PC3.

It is important to remember that the influence of $\alpha, \beta$-unsaturated part of cinnamic acid and its derivatives relative to biological activity, was studied ${ }^{34}$ when comparing compounds (E)-3-(3,4-dihydroxyphenyl)acrylate $(\alpha, \beta$-unsaturated) and 3-methyl-(3,4-dihydroxyphenyl) propanoate $(\alpha, \beta$-saturated). It was observed that the compound $\alpha, \beta$-unsaturated contributes positively to the action against breast cancer cells (T-47D) and colon (WiDr), with $\mathrm{IC}_{50}$ values 64 and $59 \mu \mathrm{M}$, respectively, while the saturated compound is inactive. On the other hand, in their studies Sova et al. ${ }^{35}$ compared the inhibition effect in relation to the phenol/alcohol part of the ester. The $(E)$-phenyl cinnamate derivative inhibited the growth of HeLa (cervical adenocarcinoma), K562 (myeloid leukemia), Fem-x (malignant melanoma) and MCF-7 (breast cancer) cell lines, with an $\mathrm{IC}_{50}$ of $75.6 \pm 12,52.6 \pm 3$, $69.0 \pm 4$ and $58.6 \pm 4 \mu \mathrm{M}$, respectively, presenting cytotoxic effects superior to the $(E)$-cyclohexyl cinnamate derivative, with $\mathrm{IC}_{50}>180 \mu \mathrm{M}$ in all cells. ${ }^{35}$

Analyzing the results from the point of view of the structures of the products, there was not a sufficiently coherent answer, however, allows some considerations. For example, 7 showed toxicity $<50 \%$ on the NCI scale, while its analog $\mathbf{1 5}$, with a methoxy group in the para position on the cinnamate part and with toxicity $>50 \%$ on the same scale, exhibited very low $\mathrm{IC}_{50}$ against all cell lines. In another example, 4 exhibited toxicity $<50 \%$ on the NCI scale, while its $\mathbf{1 2}$ analogue, with a methoxy group in the para position in the cinnamate part, affected SNB-19 and PC3 cells growth with $\mathrm{IC}_{50}$ values of 42.1 and $41.9 \mu \mathrm{M}$, respectively, and showed a potent antiproliferative effect against HTC-116 cells with an $\mathrm{IC}_{50}$ value of $16 \mu \mathrm{M}$. Finally, 18 showed remarkable toxicity to HTC-116 cells 
$\left(\mathrm{IC}_{50} 15 \mu \mathrm{M}\right.$ ), whereas 17 with a similar structure (inversion of methyl and isopropyl substituents in the aromatic ring of the phenol part) was not even detectable for $\mathrm{IC}_{50}$.

Studies involving cinnamic acids and analgesics have shown that inhibition targets in several cancer cell lines occur through the inhibitory action on the deoxyribonucleic acid (DNA) synthesis of growing cells. ${ }^{36}$ In general, the data indicate that cinnamates inhibit cell growth by selective induction of cell death and cycle disruption. ${ }^{37-39}$ Thus, the inhibitory action of compounds that showed activity in front of cancer cells in this work, was also suspected of involving inhibition in the synthesis of DNA and guaranteeing an interruption of the cell cycle.

\section{Conclusions}

In this study, eighteen esters were obtained through the Stiglich esterification. The MTT test shows that the activities of compounds with an aromatic ring in the cinnamoyl fraction are more active than cyclohexyl. In comparison between the esters obtained, this study showed that the compound $\mathbf{1 2}$ is the most potent against HCT$116, \mathrm{PC} 3$ and SNB-19 cells, with the lowest $\mathrm{IC}_{50}$ value of $16.2 \mu \mathrm{M}$ in the HCT-116 strain. The compound $\mathbf{1 8}$ also has a low $\mathrm{IC}_{50}$ value in HCT-116 $(15.38 \mu \mathrm{M})$. The compound 8 was the only one that showed the highest cytotoxicity in HL60 $\left(\mathrm{IC}_{50}=25.2 \mu \mathrm{M}\right)$. The compounds $\mathbf{8 ,}, \mathbf{1 2}$ and $\mathbf{1 8}$ showed selectivity against normal cells (L929). According to some examples observed, there was an apparent increase in biological activity with increased conjugation in the cinnamate fraction, provided by electron donating substituents, such as methoxy and hydroxyl groups. This research indicates that the tested cinnamic acid derivatives present good initial performance for the development of candidates for antineoplastic drugs, bringing new perspectives for the structurally modified natural substances under study, contributing to the knowledge and elaboration of new bioactive compounds, more effective against cancer.

\section{Supplementary Information}

Supplementary information $\left({ }^{1} \mathrm{H}\right.$ and ${ }^{13} \mathrm{C}$ NMR, IR, HRMS and potential for cell inhibition) is available free of charge at http://jbcs.sbq.org.br, as PDF file.

\section{Acknowledgments}

This work was funded by the Conselho Nacional de Desenvolvimento Científico e Tecnológico (CNPq) and the Coordenação de Aperfeiçoamento de Pessoal de Nível Superior (CAPES).

\section{Author Contributions}

R. O. G. was responsible for project administration, conceptualization, analysis, investigation, methodology and writing original draft; I. F. F. for responsible for the characterization experiments; M. F. S. S. for cytotoxicity experimental methodology; C. Ó. P. to supervise and conceptualization cytotoxicity experiments; G. J. Z. for investigation; D. Z. for conceptualization, project administration, writing-review and editing; T. L. G. L. for conceptualization, project administration; writing-review and editing; F. J. Q. M. analyzed the experimental results, revised the manuscript, writing-review and editing.

\section{References}

1. World Health Organization (WHO); https://www.who.int/en/ news-room/fact-sheets/detail/cancer, accessed on February 11, 2020.

2. Ali, I.; Rahis-ud-din; Saleem, K.; Aboul-Enein, H. Y.; Rather, A.; Cancer Ther. 2011, 8, 6.

3. Yuan, H.; Ma, Q.; Ye, L.; Piao, G.; Molecules 2016, 21, 559.

4. Stierle, A.; J. Nat. Prod. 2018, 81, 1125.

5. Kumar, N.; Pruthi, V.; Biotechnol. Rep. 2014, 4, 86.

6. Adisakwattana, S.; Nutrients 2017, 2, 16.

7. Mancuso, C.; Santangelo, R.; Food Chem. Toxicol. 2014, 65, 185.

8. Filho, A. C. V. A.; Rodrigues, P. A. S.; Benjamin, S. R.; Paim, R. T. T.; Holanda, M. O.; Silva, J. Y. G.; Milo, T. S.; Vieira, I. G. P.; Queiroz, M. G. R.; Guedes, M. I. F.; Environ. Toxicol. Pharmacol. 2017, 56, 198.

9. Rodrigues, P. A. S.; Guedes, F. I.; Marques, M. M. M.; Silva, I. N. G.; Vieira, I. G. P.; Int. J. Pharm. Pharm. Sci. 2014, 6, 115.

10. Gießel, J. M.; Serbian, I.; Loesche, A.; Csuk, R.; Bioorg. Chem. 2019, 90, 103058.

11. Sova, M.; Mini-Rev. Med. Chem. 2012, 12, 749.

12. Malheiro, J. F.; Maillard, J. Y.; Borges, F.; Simões, M.; Int. Biodeterior. Biodegrad. 2019, 141, 71.

13. Seck, R.; Mansaly, M.; Gassama, A.; Cavé, C.; Cojean, S.; J. Chem. Pharm. Res. 2018, 10, 1, available at https://www. jocpr.com/articles/synthesis-and-antimalarial-activity-ofcinnamic-acid-derivatives.pdf, accessed in June 2021.

14. Lima, T. C.; Ferreira, A. R.; Silva, D. F.; Lima, E. O.; de Sousa, D. P.; Nat. Prod. Res. 2018, 32, 572.

15. Xu, C. C.; Deng, T.; Fan, M. L.; Lv, W. B.; Liu, J. H.; Yu, B. Y.; Eur. J. Med. Chem. 2016, 107, 192.

16. Chu, F.; Zhang, W.; Guo, W.; Wang, Z.; Yang, Y.; Zhang, X.; Fang, K.; Yan, M.; Wang, P.; Lei, H.; Molecules 2018, 23, 322.

17. Lutjen, A. B.; Quirk, M. A.; Barbera, A. M.; Kolonko, E. M.; Bioorg. Med. Chem. 2018, 26, 5291. 
18. Shirahata, T.; Nagai, T.; Hirata, N.; Yokoyama, M.; Katsumi, T.; Konishi, N.; Nishino, T.; Makino, K.; Yamada, H.; Kaji, E.; Kiyohara, H.; Kobayashi, Y.; Bioorg. Med. Chem. 2017, 25, 1747.

19. Neises, B.; Steglich, W.; Angew. Chem., Int. Ed. 1978, 17, 522.

20. Tharamak, S.; Yooboon, T.; Pengsook, A.; Ratwatthananon, A.; Kumrungsee, N.; Bullangpoti, V.; Pluempanupat, W.; Pest Manage. Sci. 2020, 76, 928.

21. Nikumbh, V. P.; Tare, V. S.; Mahulikar, P. P.; J. Sci. Ind. Res. 2003, 62, 1086.

22. Foote, P. A.; J. Am. Pharm. Assoc. 1928, 17, 958.

23. Dikusar, E. A.; Kozlov, N. G.; J. Org. Chem. 2005, 41, 992.

24. Tawata, S.; Taira, S.; Kobamoto, N.; Zhu, J.; Ishihara, M.; Toyama, S.; Biosci., Biotechnol., Biochem. 1996, 60, 909.

25. Mosmann, T.; J. Immunol. Methods 1983, 65, 55.

26. Lima, B. A. V.; Corrêa, R. S.; Graminha, A. E.; Varela Jr., J. J. G.; da Silva, A. B. F.; Ellena, J.; Silva, T. E. M.; Batista, A. A.; J. Braz. Chem. Soc. 2020, 31, 1352.

27. David, C. C.; Lins, A. C. S.; Silva, T. M. S.; Campos, J. F.; Silva, T. G.; Militão, G. C. G.; Camara, C. A.; J. Braz. Chem. Soc. 2019, 30, 8.

28. Hostettmann, K.; Methods in Plant Biochemistry; Academic Press: London, 1991.

29. GraphPad Prism, version 5.0; GraphPad Software, Inc., San Diego, USA, 2007.

30. Silverstein, R. M.; Webstewr, F. X.; Kiemle, D. J.; Identificação de Compostos Orgânicos; LTC Editora: Rio de Janeiro, 2006.
31. Skehan, P.; Storeng, R.; Scudiero, D.; Monks, A.; Mcmahon, J.; Vistica, D.; Warren, J. T.; Bokesch, H.; Kenney, S.; Boyd, M. R.; J. Natl. Cancer Inst. 1990, 82, 1107.

32. Guedes, J. A. C.; Filho, E. G. A.; Rodrigues, T. H. S.; Silva, M. F. S.; Souza, F. V. D.; Alexandre, L. M. S.; Alves, R. E.; Canuto, K. M.; Brito, E. S.; Pessoa, C. Ó.; Nascimento, R. F.; Zocolo, G. J.; Ind. Crops Prod. 2018, 124, 466.

33. Souza, L. G. D. S.; Almeida, M. C. S.; Lemos, T. L. G.; Ribeiro, P. R. V.; Brito, E. S.; Silva, V. L. M.; Silva, A. M. S.; Braz-Filho, R.; Costa, J. G. M.; Rodrigues, F. F. G.; Barreto, F. S.; Moraes, M. O.; Bioorg. Med. Chem. Lett. 2016, 26, 435.

34. Reta, G. F.; Tonn, C. E.; Ríos-Luci, C.; León, L. G.; Pérez-Roth, E.; Padrón, J. M.; Donadela, O. J.; Nat. Prod. Commun. 2012, 10, 1341 .

35. Sova, M.; Zizak, Z.; Stankovic, J.; Prijatelj, M.; Turk, S.; Juranic, Z.; Mlinaric-Rascan, I.; Gobec, S.; Med. Chem. 2013, 9, 633.

36. Niero, E. L. O.; Machado-Santelli, G. M.; J. Exp. Clin. Cancer Res. 2013, 32, 31.

37. Imai, M.; Yokoe, H.; Tsubuki, M.; Takahashi, N.; Biol. Pharm. Bull. 2019, 42, 1134.

38. Almeer, R. S.; Aref, A. M.; Hussein, R. A.; Othman, M. S.; Abdel, M. A. E.; Anticancer Agents Med. Chem. 2018, 19, 356.

39. Uesawa, Y.; Sakagami, H.; Okudaira, N.; Toda, K.; Takao, K.; Kagaya, H.; Sugita, Y.; Anticancer Res. 2018, 38, 817.

Submitted: April 10, 2021 Published online: June 11, 2021 